\title{
Relations Among Parentification, Parenting Beliefs, and Parenting Behaviors
}

\author{
Brittney T. Black ${ }^{\mathrm{a}} \&$ Merry J. Sleigh ${ }^{\mathrm{a}}$
}

\begin{abstract}
Instrumental parentification occurs when children take on adult chores, and emotional parentification occurs when children take the responsibility for providing an adult-level of emotional support. This study examined how the level of parentification and perceived unfairness in the childhood home affected adults' parenting beliefs and future parenting behaviors. One hundred and seven young adults responded to the Parentification Questionnaire which assessed levels of instrumental parentification, emotional parentification, and perceived fairness. Participants were asked to imagine themselves as parents and then respond to questions that assessed their parenting beliefs, planned parenting behaviors, and demographics. Results revealed that emotional parentification and perceived unfairness were related to adults' decreased self-esteem and feelings of attractiveness. In addition, parentification and perceived unfairness in the childhood home were linked to negative feelings toward participants' own parents; however, parentification did not clearly predict factors associated with parenting.
\end{abstract}

Keywords: Parentification; Parenting; Behaviors; Beliefs

\section{Introduction}

Parentification occurs when children are required to function as an adult when it is developmentally inappropriate for them to do so (Wells \& Jones, 2000). The practice of parentification has been occurring for centuries but only within the last few decades have researchers associated a name with it, and research on this topic is limited. There are two types of parentification: emotional and instrumental. Emotional parentification occurs when children provide emotional support to other family members, such as being treated as a parent's friend and confidant (Jurkovic, Thirkfield \& Morrell, 2001). Instrumental parentification occurs when children take on family tasks that are typically done by adults, such as grocery shopping or caring for siblings (Jurkovic et al, 2001). Parentification often co-occurs with a sense that the home environment is unfair to them, termed 'perceptions of fairness' (Jurkovic et al, 2001). This phenomenon is seen across cultures (e.g., Shih, Wu, \& Lin, 2010; Titzmann, 2012) and has been linked to both negative and positive outcomes in adulthood (Carroll \& Robinson, 2000; Hooper, Marotta, \& Lanthier 2008; Jurkovic et al, 2001; Peris, Goeke-Morey, Cummings, \& Emery, 2008; Wells \& Jones, 2000). For example, adults who experienced parentification during childhood have an increased likelihood of engaging in substance abuse (Hooper, DeCoster, White \& Voltz, 2011), but also are likely to exhibit resilience in the face of negative events (Hooper, Marotta, \& Lanthier 2008). The goal of the current study was to further our understanding of this relatively new construct by examining whether the childhood experience of parentification relates to adults' beliefs about their own parenting.

\section{Literature Review}

Parentification has been associated with several factors that are believed to be causal. Parentification has been consistently linked to family unpredictability, parental alcoholism, poor parental health, marital conflict, and low family income (Burnett, Jones, Bliwise, \& Ross, 2006;
Burton, 2007; Godsall, Jurkovic, Emshoff, Anderson, \& Stanwyck, 2004; Kelley et al, 2007; Hooper, Doehler, Jankowski, \& Tomek, 2012; Peris et al, 2008). Not only are there many factors that may lead to parentification, these factors can have differential impacts. Carroll and Robinson (2000) found that children of workaholics and children of alcoholics both had the potential for parentification; however, the children of workaholics experienced significantly higher levels of parentification than children of alcoholics.

In addition to being multi-causal, parentification is linked to multiple adverse outcomes. Parentification has been linked to a variety of pathologies such as eating disorders, mood disorders, substance abuse disorders, and personality disorders (Hooper, DeCoster, White, \& Voltz, 2011). In addition, children who experience parentification are vulnerable to the imposter phenomenon, as described by Castro, Jones, and Mirsalimi (2004). The imposter phenomenon occurs when individuals feel as though people's positive perceptions of them are inaccurate, resulting in a lack of confidence in one's uniqueness, talent, and the ability to meet others' demands (Castro et al, 2004). Adults who experienced parentification as a child are also vulnerable to feelings of shame versus guilt (Wells \& Jones, 2000). Shame is considered the more damaging of the two, because mistakes leave a shame-prone person feeling badly about the self; in contrast, guilt is a focus on the behavior and ways to correct it.

Parentification may have more of an impact than parents realize. Peris, Goeke-Morey, Cummings, and Emery (2008) examined parental reliance on emotional support from children during marital conflicts. The researchers found that mothers tended to seek emotional support from children more than fathers and especially from their daughters. The results also showed that parents reported more closeness and warmth with their children, while children reported low levels of warmth and support from parents. These findings suggest that children feel more negative effects from emotional parentification during marital conflict than parents perceive. 
Despite the many negative associations, previous research has revealed some positive associations with parentification. Hooper, Marotta, and Lanthier (2008) found that people who experienced parentification could have posttraumatic growth, particularly increased resiliency. Posttraumatic growth refers to the positive outcome that the individual experiences after going through an aversive situation. In Hooper et. al's (2008) case, the aversive situation was parentification and as a result of experiencing parentification, the individual was more resilient to negative life events. Similarly, instrumental parentification in the form of caring for siblings was linked to psychosocial adjustment (versus maladjustment) perhaps because the act of caregiving increased self-efficacy in the individual (Fitzgerald, Schneider, Zinzow, Jackson, \& Fossel, 2008). The potential for positive outcomes may depend to some extent on the child. For example, Williams and Francis (2010) argued that an internal locus of control may shield children from the negative effects of parentification. Despite the potential for positive outcomes, researchers who examine both typically find more negative effects of parentification than positive effects, especially in regard to emotional parentification (Black, Lindberg, Garansi, \& Sleigh, 2011). Similarly, Hooper, Doehler, Jankowski and Tomek (2012) found that adolescents with alcoholic parents were less likely to have alcohol problems themselves if they were experiencing parentification, but were more likely to exhibit depressive symptoms. In other words, parentification was a buffer for one adverse outcome and a moderator for the other.

Because parentification is so closely tied with parenting, it seems logical that childhood parentification would impact adults' parenting behaviors. Hooper (2007) suggested that parentification should be conceptualized as interference with healthy attachment between the parent and child. Emotional parentification and perceived unfairness may be particularly detrimental to the parent-child relationship, as they both contain an emotional element. In fact, Nutall, Valentino, and Borkowski (2012) recently found that mothers who experienced parentification were less warm with their young children, resulting in those children exhibiting more problematic externalizing behaviors. Instrumental parentification, in contrast, is more about practical matters, such as getting dinner on the table and has even been linked to increased interpersonal competence (Thirkield, 2002).

We examined how childhood parentification related to adults' parenting beliefs and behaviors. We hypothesized that: (1) participants who experienced parentification would perceive their own parents in a poor light and report a desire to raise their own children in a different way and (2) adults who experienced parentification during their childhood would have different parenting behaviors and beliefs than adults who had not experienced parentification. Our hypothesis was nondirectional because we were unsure of whether people would repeat their experiences with childhood parentification or they would choose a different parenting experience than their own.

\section{Method}

\section{Participants}

One hundred seven adults with a mean age of 20.63 ( $s d$ $=2.75)$ participated in the study. Forty-seven percent identified as Caucasian, $40 \%$ identified as African-American, and $13 \%$ identified as another ethnicity. Sixty-six percent of the participants were women and $34 \%$ were men. Participants confirmed their status as non-parents. This study was approved by the university's Institutional Review Board, and all participation was voluntary.

\section{Materials}

We measured instrumental and emotional parentification, and perceived fairness in participants' childhood homes with a modified Parentification Questionnaire (Hooper \& Wallace, 2010). The original Parentification Questionnaire was a 30 item, self-report measure developed by Jurkovic and Thirkield (1998). Hooper and Wallace (2010) focused on 21-items that loaded on three constructs (instrumental parentification, emotional parentification, and perceived fairness), and demonstrated this modified scale as a reliable measure of parentification; Cronbach's alpha coefficients were over. 80 for all scales (instrumental parentification $=.81$, emotional parentification $=.82$, perceived unfairness $=.88$ ).

Instrumental parentification included five items such as, "I often did the family's laundry" and "My parents expected me to help discipline my siblings." Emotional parentification included seven items such as, "At times I felt I was the only one my mother or father could turn to" and "In my family I often made sacrifices that went unnoticed." Perceived fairness included nine items such as, "My parents often tried to get me to take their sides in conflicts" and "It often seemed that my feelings weren't taken into account in my family." Participants rated how true each statement was of them on a five-point Likert scale with one representing "Strongly Disagree" and five representing "Strongly Agree." Some items were reverse-scored and a mean score was calculated for each sub-scale (Hooper \& Wallace, 2010). The higher the score on each sub-scale, the more of that specific type of parentification the participants experienced in their childhood homes.

Thirty-five additional questions/statements were created by the researchers. Parenting behaviors were assessed by having participants respond with their level of agreement to five statements such as "I try to protect my child from growing up too fast" and "I probably do more for my child than I should." Parenting beliefs were assessed by having participants respond with their level of agreement to 15 statements such as "Children deserve an allowance" and "Children should not question their parents." The parenting belief and behavior statements were created after examining existing lists of parenting activities and seeing many consistent themes across them. We also asked participants to rank three future behaviors (caring for your spouse/partner, caring for your child/children, having a successful career) from the one most important to the one least important to them. Last, participants responded to demographic questions.

\section{Procedure}

The survey was posted online through surveymonkey. Participants were recruited for the study through social media using a snowball technique. The authors posted the link to the survey on their own social media sites, and visitors to those sites were asked to share the link through their social media sites. Participants also were provided the link through undergraduate classrooms and offered extra credit for participating. The survey was posted for a period of 
two months, and participants recruited outside of the classroom were offered no incentives for participation.

Participants first responded to the Parentification Questionnaire, followed by the questions related to parenting behaviors and beliefs. Participants were asked to imagine themselves in the role of a parent and then to respond in a way that reflected the parenting beliefs and behaviors they planned to use with their own child(ren). Data was analyzed using Pearson's correlations.

\section{Results}

We computed a "Perceptions of Fairness" score (POF), an "Emotional Parentification" score (EPS), and an "Instrumental Parentification" score (IPS) for each participant by following Hooper and Wallace's (2010) instructions to reverse score specified items and then calculate a mean for the relevant questions on each dimension. A higher POF score indicated that participants perceived a greater sense of unfairness in their childhood home. For IPS and EPS, a higher score indicated more experience with that particular type of parentification.

We examined relationships among the sub-scales using Pearson's correlations. Emotional parentification and instrumental parentification were positively correlated, $r(99)$ $=.48, p<.01$. POF was positively correlated with both instrumental, $r(99)=.33, p<.01$ and emotional parentification, $r(102)=.66, p<.01$. These correlations, revealing medium to large effect sizes, among the sub-scales are consistent with previous research (e.g., Hooper \& Wallace, 2010).

\section{Parentification and Perceptions of Parents}

Results can be seen in Table 1. The higher the IPS, the more participants agreed that they were unhappy with the way they were raised, $r(99)=.23, p=.023$, revealing a small effect. The higher the EPS, the more participants agreed that their parents were not there for them, $r(99)=.48$, the more unhappy participants felt about the way they were raised, $r(99)=.45$, the less participants agreed that they want to raise their families in the way they were raised, $r(99)=-.49$, and the less participants agreed that their parents did the best job they could in raising them, $r(97)=-.40(p<.01$ and revealing medium effect sizes in all cases).

The higher the POF, the more participants agreed that their parents were not there for them, $r(99)=.70$, the more unhappy participants felt about the way they were raised, $r(99)=.72$, the less participants agreed that they want to raise their families in the way they were raised, $r(99)=-.71$, and the less participants agreed that their parents did the best job they could in raising them, $r(97)=-.64(p<.01$ and revealing large effect sizes in all cases).

Table 1

Correlations between parentification sub-scales and perception of parents

\begin{tabular}{|c|c|c|c|}
\hline Statement & $\overline{\text { POF }}$ & EPS & IPS \\
\hline $\begin{array}{l}\text { I want to raise my family in } \\
\text { the same way that I was } \\
\text { raised. }\end{array}$ & $\begin{array}{l}-.713 \\
.000\end{array}$ & $\begin{array}{l}-.487 \\
.000\end{array}$ & \\
\hline $\begin{array}{l}\text { Overall, I fell very } \\
\text { negative/unhappy about the } \\
\text { way in which I was raised. }\end{array}$ & $\begin{array}{l}.720 \\
.000\end{array}$ & $\begin{array}{l}.446 \\
.000\end{array}$ & $\begin{array}{l}.229 \\
.023\end{array}$ \\
\hline $\begin{array}{l}\text { I believe that my parents } \\
\text { did the best job they could } \\
\text { in raising me. }\end{array}$ & $\begin{array}{l}-.642 \\
.000\end{array}$ & $\begin{array}{l}-.395 \\
.000\end{array}$ & \\
\hline $\begin{array}{l}\text { My parents were not there } \\
\text { for me as much as I would } \\
\text { have liked them to be. }\end{array}$ & $\begin{array}{l}.690 \\
.000\end{array}$ & $\begin{array}{l}.479 \\
.000\end{array}$ & \\
\hline
\end{tabular}

\section{Parentification and Parenting Behaviors}

We examined relations among variables using Pearson's correlations. Instrumental parentification, emotional parentification, and perceived unfairness scores did not predict how participants responded to the list of parenting behaviors. In other words, a childhood experience with parentification was not linked to a particular pattern of participants' planned parenting behaviors. However we did find that participants high in POF, ranked their career as more important than caring for their spouse or children, $r(94)=-$ $.23, p<.05$, revealing a small effect size.

\section{Parentification and Parenting Beliefs}

The higher the EPS, the less participants agreed that it is very important for children to do well in school, $r(96)=-.21$, $p<.05$. The higher the POF, the less participants agreed with the notion that children should not question parents, $r(96)=-$ .24. POF was positively correlated with the belief that parents should respect their child's privacy, $r(96)=.21$, and that parents should always speak in a calm voice to their children, $r(96)=.24$ ( $p<.05$ in all cases, revealing small effect sizes). IPS did not predict participants' parenting beliefs.

Because we did not find strong relations between childhood experiences and parenting behaviors, we examined participants' overall responses to parenting situations. The results revealed that participants varied in their beliefs about parenting. Over $50 \%$ of participants were in agreement on only nine of the 15 parenting statements (see Table 2). 
Table 2

Percentage of Participants who Agreed with Parenting Belief Statements

\begin{tabular}{|c|c|}
\hline Statement & $\begin{array}{c}\text { Percentage of } \\
\text { Participants who } \\
\text { Agreed }\end{array}$ \\
\hline Parents should tell their children "no" when needed. & $98 \%$ \\
\hline Parents should clearly explain the rules and expectations to their child. & $94 \%$ \\
\hline It is very important that children do well in school. & $92 \%$ \\
\hline Physical affection with children is important. & $91 \%$ \\
\hline Parents should get their child to try new things. & $89 \%$ \\
\hline Parents should have high expectations of their children. & $75 \%$ \\
\hline Parents should be sensitive to every one of their child's moods. & $66 \%$ \\
\hline $\begin{array}{l}\text { It is important for parents to teach their children to share their beliefs } \\
\text { (religious or cultural). }\end{array}$ & $61 \%$ \\
\hline Parents should respect their child's privacy. & $58 \%$ \\
\hline Children need to compete with others to learn about the real world. & $46 \%$ \\
\hline Spanking a child is an appropriate form of discipline. & $45 \%$ \\
\hline Parents should always speak in a calm voice to their children. & $45 \%$ \\
\hline Children deserve an allowance. & $31 \%$ \\
\hline Children should not question their parents. & $24 \%$ \\
\hline It is the school's responsibility to educate children, not the parent's job. & $3 \%$ \\
\hline
\end{tabular}

\section{Post-hoc Analyses}

The higher the POF, the lower the participants' selfesteem, $r(94)=-.37, p<.01$, and the less attractive participants rated themselves, $r(95)=-.21, p=.04$, revealing small effect sizes. The higher the EPS, the lower the participants' self-esteem, $r(94)=-.27, p<.01$, and the less attractive participants rated themselves, $r(95)=-.23, p=.026$, revealing small effect sizes. IPS did not predict participants' self-esteem or perceptions of their own attractiveness.

\section{Discussion}

The purpose of the study was to examine whether childhood experiences with parentification affected adults parenting beliefs. We hypothesized that participants who experienced parentification would perceive their own parents in a poor light and report a desire to raise their own children in a different way. This hypothesis was supported. Participants high in instrumental parentification, emotional parentification, or perceived fairness reported being "unhappy" with the way in which they were raised. Emotional parentification and perceptions of fairness were also associated with participants wanting to raise their own families differently than they way in which they were raised. In other words, childhood parentification was linked to adults' unhappiness with their childhood home, as well as a general desire to avoid replicating that situation with their own children.

We also hypothesized that parentification would influence the way adults planned to parent, and this hypothesis seemed especially likely to be met given the unhappiness that our parentified participants reported with their own parents; however, our results only partially supported this hypothesis. Emotional parentification, perceived fairness, and instrumental parentification levels did not predict participants' support of specific parenting behaviors.

Similarly, experience with emotional parentification and perceptions of fairness predicted limited parenting beliefs. Participants with high EPS were less likely to agree that it was important for children to do well in school. Participants with high POF disagreed that children should not question their parents and agreed that parents should respect children's privacy and speak to children calmly. Participants who felt 
they were treated unfairly seem to be revealing parenting beliefs that decrease the power of the parent over the child and thus, decrease the ability for unfair treatment. Participants who scored high in POF also ranked career as a more important priority than caring for their spouse of children. Once again, these participants may be attempting to compensate for the unfairness they perceived in their childhood home, as lower family income is one predictor of parentification (Burton, 2007).

Despite these associations, it is noteworthy that most parenting beliefs did not relate to experience with parentification. For example, instrumental parentification was not associated with parenting beliefs. Out of the fifteen presented parenting beliefs, emotional parentification was associated with only one belief, and perceived fairness was associated with three beliefs. One interpretation is that an individual's experiences with childhood parentification do not relate to their planned parenting in adulthood. Another plausible interpretation is that participants who experienced parentification wanted to raise their own families differently but did not share a common idea of what this might mean. In other words, participants may plan to be different than their own parents in a variety of ways, with some planning to be more permissive and others planning to be more hands-on. In support of this interpretation, we did not find clear consensus in how participants responded to the parenting behavior statements.

Taylor, Lauder, Moy and Corlett (2009) argued that even though the details might be complicated, there is general agreement among adults about what it means to be a "good enough" parent. In other words, most young adults share an implicit idea of how good parents function. Interestingly, our participants who experienced parentification did not show this expected agreement. This lack of agreement may reflect the fact that children experiencing parentification do not have enough exposure to typical parenting situations that lead to normative parenting ideas. Burton (2007) demonstrated that children who experience parentification are often perceived as "different" from other children because of the uniqueness of their life experiences. Another possibility is that this finding reflects yet another atypical outcome of parentification not yet identified in previous research.

Previous research revealed that sometimes individual's parenting beliefs and behaviors do not match (Burchinal, Skinner, \& Reznick, 2010). In this study, we were asking nonparents to predict their future parenting behaviors. Although parentified participants shared the belief that they did not want to be like their own parents, they may not have been able to accurately translate that belief into accurate predictions of their own parenting, resulting in the diversity of responses we collected. Perhaps if participants were actually in a parenting situation, we would have uncovered unique patterns of behavior as did Nutall, Valentino, and Borkowski (2012).

Our results also revealed that emotional parentification and perceived unfairness predicted participants' decreased self-esteem and perceived attractiveness, matching previous research (Black et al, 2011). In other words, experiences with parentification can have long-lasting effects on feelings of self-worth. This finding raises questions about how parentification can impact people's self-image without having any clear effect on their image of themselves as parents. This area is ripe for future research.

One limitation of the current study is that we studied only non-parents. Future studies may want to focus specifically on people who are actually parents and compare parenting beliefs and behaviors of non-parents to actual parents. Future researchers also could use observational methodology to compare parenting behaviors of adults who experienced parentification and those who did not, as well as to assess how their parenting beliefs and behaviors match.

Another limitation is that we did not randomly sample our participants from a broad population, relying instead on a university population. Because of their successful enrollment at a university, these participants may not have experienced extreme parentification, such as that of adult children of alcoholics. Adults in more extreme childhood situations may have had less opportunity to witness typical parenting and may exhibit more extreme reactions to their childhood home. That greater impact may translate into unique parenting beliefs. Future studies may want to focus on more vulnerable populations.

In sum, this data in combination with previous research, demonstrates that parentification impacts individuals in ways that are likely to influence their parenting, such as decreased self-esteem. However, parentification did not clearly predict parenting beliefs or behaviors. Adults who experienced childhood parentification expressed unhappiness with the way they were raised, yet did not report consensus in the ways they planned to differ from their own parents. This finding adds to our growing understanding of parentification and parenting.

\section{References}

Black, B., Lindberg, K., Garansi, A. \& Sleigh, M. J. (2011). Parentification implications: A study on positive and negative effects of childhood parentification. Presented at the Southeastern Psychological Association conference, New Orleans, LA.

Burchinal, M., Skinner, D., \& Reznick, J.S. (2010). European American and African American mothers' beliefs about parenting and disciplining infants: A mixedmethod analysis. Parenting: Science \& Practice, 10(2), 79-96.

Burnett, G., Jones, R., Bliwise, N.G., \& Ross, L.T. (2006). Family unpredictability, parental alcoholism, and the development of parentification. American Journal of Family Therapy, 34, 181-189.

Burton, L. (2007). Childhood adultification in economically disadvantaged families: A conceptual model. Family Relations, 56, 329-345.

Carroll, J.J., \& Robinson, B.E. (2000). Depression and parentification among adults as related to parental workaholism and alcoholism. The Family Journal: Counseling and Therapy for Couples and Families, $8,360-367$.

Castro, D.M., Jones, R.A., \& Mirsalimi, H. (2004). Parentification and the imposter phenomenon: An empirical investigation. American Journal of Family Therapy, 32, 205-216.

Fitzgerald, M.M, Schneider, R.A, Zinzow, H.M., Jackson, J., \& Fossel, R.V. (2008). Child sexual abuse, early 
risk, and childhood parentification: Pathways to current psychosocial adjustment. Journal of Family Psychology, 22, 320-324.

Godsall, R.E., Jurkovic, G.J., Emshoff, J., Anderson, L., \& Stanwyck, D. (2004). Why some kids do well in bad situations: Relation of parental alcohol misuse and parentification to children's self-concept. Substance Use and Misuse, 39, 789-809.

Hooper, L.M. (2007). The application of attachment theory and family systems theory to the phenomena of parentification. The Family Journal, 15(3), 217223.

Hooper, L. M., DeCoster, J., White, N., \& Voltz, M. L. (2011). Characterizing the magnitude of the relation between self-reported childhood parentification and adult psychopathology: A meta-analysis. Journal of Clinical Psychology, 67, 1-16.

Hooper, L. M., Doehler, K., Jankowski, P. T., \& Tomek, S. E. (2012). Patterns of self-reported alcohol use, depressive symptoms, and body mass index in a family sample: The buffering effects of parentification. The Family Journal, 20, 164-178.

Hooper, L.M., Marotta, S.A, \& Lantheir, R.P. (2008). Predictors of growth and distress following childhood parentification: A retrospective exploratory study. Journal of Child and Family Studies, 17(5), 693-705.

Hooper, L.M., \& Wallace, S.A. (2010). Evaluating the parentification questionnaire:

Psychometric properties and psychopathology correlates. Contemporary Family Therapy: An International Journal, 32(1), 52-68.

Jurkovic, G.J., \& Thirkield, A. (1998). Parentification Questionnaire (Available from G.J.

Jurkovic, Department of Psychology, Georgia State University, University Plaza, Atlanta, GA 30303).

Jurkovic, G. J., Thirkfield, A., \& Morrell, R. (2001). Parentification of adult children of divorce: A multidimensional analysis. Journal of Youth and Adolescence, 30, 245-257.
Kelley, M.L., French, A., Bountress, K., Keefe, H.A., Schroeder, V., Steer, K., Fals-Stewart, W., \& Gumienny, L. (2007). Parentification and family responsibility in the family of origin of adult children of alcoholics. Addictive Behaviors, 32, 675-685.

Nuttall, A.K., Valentino, K., \& Borkowski, J.G. (2012). Maternal history of Parentification, maternal warm responsiveness, and children's externalizing behavior. Journal of Family

Psychology, 26(5).

Peris, T.S., Goeke-Morey, M.C., Cummings, E.M., \& Emery, R.E. (2008). Martial conflict and support seeking by parents in adolescence: Empirical support for the parentification construct. Journal of Family Psychology, 22(4), 633-642.

Shih, F.M., Wu, L.C., \& Lin, S.H. (2010). A correlational study on parentification, self-differentiation, and health for students in senior high and vocational high school in Taiwan. Bulletin of Educational Psychology, 41(4), 823-846.

Taylor, J., Lauder, W., Moy, M., \& Corlett, J. (2009). Practitioner assessments of 'good enough' parenting: Factorial survey. Journal of Clinical Nursing, 18(8), 1180-1189.

Thirkield, A. (2002). The role of fairness in emotional and social outcomes of childhood filial responsibility. Unpublished doctoral dissertation, Georgia State University, Atlanta, GA.

Titzmann, P.F. (2012). Growing up too soon? Parentification among immigrant and native adolescents in Germany. Journal of Youth and Adolescence, 41(7), 880-893.

Wells, M., \& Jones, R. (2000). Childhood parentification and shame-proneness: A preliminary study. American Journal of Family Therapy, 28(1), 19-27.

Williams, K., \& Francis, S.E. (2010). Parentification and psychological adjustment: Locus of control as a moderating variable. Contemporary Family Therapy: An International Journal, 32(3), 231-237. 